\title{
Producción de melón (Cucumis melo L.) en invernadero: efecto de poda y densidad de siembra
}

\section{Melon (Cucumis melo L.) production under greenhouse conditions: effect of pruning and plant density}

\author{
Jorge Manuel Díaz-Alvarado ${ }^{1}$ \\ José Eladio Monge-Pérez ${ }^{2}$ \\ Universidad de Costa Rica, Costa Rica
}

\begin{abstract}
Resumen. Se evaluó el efecto de dos densidades de siembra (1,5 y 2,0 plantas $\left./ \mathrm{m}^{2}\right)$ y dos tipos de poda (un tallo secundario y dos tallos secundarios) sobre el rendimiento y la calidad del melón tipo Amarillo JMX-904 F-1 (Cucumis melo L. var. inodorus), en Alajuela, Costa Rica, entre marzo y julio de 2011. No se presentaron diferencias estadísticamente significativas entre tratamientos para el número total de frutos por planta, el peso promedio del fruto, el porcentaje de sólidos solubles totales, la firmeza de la pulpa, y la relación pulpa:cavidad del fruto. Se presentaron diferencias estadísticamente significativas entre tratamientos para el rendimiento total; el mejor tratamiento correspondió a plantas con poda a un tallo secundario y con una densidad de siembra de 2,0 plantas $/ \mathrm{m}^{2}$, el cual produjo un rendimiento total de 27,5 ton/ha.
\end{abstract}

Palabras clave. Melón amarillo, poda, densidad de siembra, rendimiento, calidad

\begin{abstract}
An evaluation of the effect of two planting densities (1.5 and $2.0 \mathrm{plants} / \mathrm{m}^{2}$ ) and two pruning methods (one secondary shoot and two secondary shoots) on the yield and quality of Yellow Honey Dew melon JMX-904 F-1 (Cucumis melo L. var. inodorus), was performed in Alajuela, Costa Rica, from March to July 2011. There were no statistically significant differences between treatments for the total number of fruits per plant, mean fruit weight, the percentage of total soluble solids, fruit pulp firmness, and the fruit pulp: cavity ratio. There were statistically significant differences between treatments for total yield; the best treatment was one secondary shoot pruning and 2.0 plants $/ \mathrm{m}^{2}$, with a totalyield of 27.5 ton/ha.
\end{abstract}

Keywords. Yellow Honey Dew melon, pruning, planting density, yield, quality 


\section{Introducción}

El melón, Cucumis melo L. es una especie que pertenece a la familia Cucurbitaceae, y es originaria del África tropical. Este cultivo es muy apetecido por sus frutos, aunque también se consumen sus semillas, hojas y flores (National Research Council, 2008; FAO, 2012). Se trata de una planta herbácea, anual, con la característica de que los tallos pueden ser rastreros o trepadores si se les facilita un entutorado (Torres, 1997; Reche, 2007).

Una forma de clasificar comercialmente a los melones es según algunas características externas e internas del fruto. Los tipos de melón que se cultivan en Costa Rica son: Amarillo, Honey Dew, Charentais, Orange Flesh, Galia, Harper, Cantaloupe y Piel de Sapo (Monge-Pérez, 2014); sin embargo, existen muchos otros tipos de melón (Torres, 1997; Reche, 2007; SEPSA, 2010).

En Costa Rica, la temporada melonera está limitada a la estación seca (diciembre hasta abril), debido al alto costo y a los bajos rendimientos que se obtienen durante la época lluviosa, producto principalmente de las enfermedades fungosas que se favorecen con la humedad, en especial el mildiú velloso causado por el hongo Pseudoperonospora cubensis (Monge-Pérez, 2014). Sin embargo, esta restricción temporal no limita la relevancia del cultivo para el país, ya que durante el año 2011 fue el quinto producto agrícola de mayor importancia económica, con un monto de exportaciones de 66,9 millones de dólares, equivalente a un $2,9 \%$ del valor total de las exportaciones agrícolas para ese año (SEPSA, 2012; Autor2, 2014).

El área cultivada con melón en Costa Rica se ha reducido de forma considerable en los últimos años, llegando hasta una disminución de un 50 \% en comparación con las áreas sembradas durante los años 2005-2007, cuando se dio el máximo pico productivo en la última década.
En la actualidad, se siembran aproximadamente 5000 hectáreas. Dentro de las razones que se dan para esta disminución se citan: un exceso de oferta en los mercados internacionales, la migración en la mano de obra debida al auge del sector construcción, el incremento de los efectos negativos debido a las lluvias y a la alta humedad relativa que se han venido registrado durante la época de cultivo, y a la crisis económica del año 2008 (SEPSA, 2010; Monge-Pérez, 2014).

El uso de ambientes protegidos viene a ser una herramienta novedosa que permite el desarrollo de una agricultura competitiva e innovadora, la cual incluye una serie de tecnologías que permiten proveer un producto de excelente calidad, indispensable para poder competir en el mercado globalizado actual (Santos, Obregón-Olivas, y Salamé-Donoso, 2010). Sin embargo, en Costa Rica esta herramienta aún no ha sido utilizada extensamente para la producción de hortalizas, y no existe en la actualidad ningún sector que cultive, de manera estable, melón en invernadero.

Varias son las ventajas que podría generar el uso de ambientes protegidos para la producción local de melón, entre las cuales se pueden citar dos muy importantes: permite producir fruta durante todo el año, lo que implica la producción de melón durante la época lluviosa, el cual podría ser comercializado en el mercado local a un buen precio, o ser exportado aprovechando que nuestro país ya tiene desarrollado el mercado internacional (Santos, Obregón-Olivas, y Salamé-Donoso, 2010; Autor2, 2011), y también permite obtener mejores rendimientos en comparación con los conseguidos a campo abierto, ya que se pueden prolongar los ciclos productivos del cultivo (Reche, 2007; Santos, Obregón-Olivas, y Salamé-Donoso, 2010; Alvarado-Sánchez y Monge-Pérez, 2015).

Dadas las potenciales ventajas de producir melón fuera de temporada, y la necesidad de encontrar nuevas formas de producción para 
sobresalir en los mercados nacionales e internacionales, se considera importante desarrollar un sistema productivo para este cultivo en invernadero, adaptado a las condiciones de nuestro país, de tal manera que se le brinde al agricultor una nueva alternativa de negocios que garantice un buen rendimiento y fruta de calidad.

La razón principal que originó los sembradíos de melón en invernadero fue ofrecer fruta al mercado cuando no existiese producción al aire libre o cuando esta fuese escasa. Para lograr esta meta, se implementaron dos prácticas importantes: el uso de un sistema de amarre o entutorado y el uso de sistemas de podas (Gómez-Guillamón, Camero, y González-Fernández, 1997).

El uso de un sistema de amarre o entutorado pretende mejorar la administración de la superficie y del espacio cultivado, con el objetivo principal de aumentar la densidad de siembra y, en consecuencia, incrementar el rendimiento por área (FAO, 2002; Reche, 2007). Existen dos tipos de sistemas de amarre: por medio de cuerdas, donde la planta se guía alrededor de la cuerda; y por medio de redes de mallas, que se colocan paralelas al cultivo, y las plantas se sujetan a estas por medio de sus zarcillos (Reche, 2007).

En Croacia, otros investigadores evaluaron el efecto de la distancia de siembra sobre el rendimiento en melón (Ban, Goreta, y Borosic, 2006). Los tratamientos utilizados incluyeron el uso de cuatro densidades de siembra: 0,$44 ; 0,55 ; 0,74$ y 1,11 plantas/m2. Los resultados determinaron que con la mayor densidad de siembra se obtenía el mejor rendimiento, con la ventaja de que no existieron diferencias importantes en el peso o la calidad del fruto entre los diferentes tratamientos.

En Israel se realizó un estudio en el cual se determinó que, conforme se incrementa la densidad de siembra, también se da un aumento en el rendimiento, pero el peso del fruto tiende a ser menor (Nerson, 1999). Los tratamientos in- cluían la siembra a 0,$5 ; 2,0$ y 8,0 plantas $/ \mathrm{m} 2$. En esa ocasión, se concluyó que con una densidad de siembra de 0,5 plantas $/ \mathrm{m} 2$ se obtiene la mayor cantidad de fruta comercializable, definida como fruta con peso superior a $1000 \mathrm{~g}$, mientras que, con mayores densidades, a pesar del aumento en el rendimiento, la cantidad de fruta comercializable fue menor.

Otros autores evaluaron en Florida cuatro densidades de siembra en melón: 1,7; 2,5; 3,3 y 4,1 plantas $/ \mathrm{m}^{2}$. Los valores obtenidos indicaron que aún con una densidad de 4,1 plantas/m2 se logró un rendimiento superior a los de las otras densidades, sin afectar las características de calidad en el fruto de melón tipo Galia (Rodríguez, Shaw, y Cantliffe, 2007).

Con respecto a la implementación de los tipos de podas, lo que se pretende es adelantar la cosecha, basándose en el principio botánico de que, en el melón, las flores femeninas o hermafroditas solo aparecen en las ramas secundarias o terciarias (Torres, 1997; FAO, 2002; Reche, 2007). El objetivo principal se basa en la teoría que indica que al podar el melón se adelanta la cosecha, pues al eliminar el tallo principal se fomenta la aparición más tempranera de tallos secundarios y terciarios, siendo en estos últimos donde se genera la mayor cantidad de frutos (Reche, 1995). Existen muchos sistemas de podas en melón, pero generalmente lo que se busca es mantener un balance para disminuir el vigor vegetativo y adelantar la aparición de flores femeninas o hermafroditas. Las prácticas de poda involucran la eliminación o conservación del tallo principal, y el manejo de uno, dos o más tallos secundarios y terciarios (Reche, 2007).

Otros autores analizaron el efecto de mantener o podar el tallo principal sobre el rendimiento y las características de calidad en frutos de melón tipo Galia, sembrados en cuatro sustratos diferentes (Eltez, Tüzel, y Boztok, 1999). Los tratamientos incluían la eliminación del tallo prin- 
cipal y el manejo de un tallo secundario, y el tratamiento testigo donde no se efectuó poda. En esta ocasión, los resultados no mostraron diferencias significativas en el rendimiento entre los distintos tratamientos, pero los frutos de mayor tamaño se encontraron en el tratamiento sin poda, mientras que la mejor firmeza de pulpa se dio en los frutos provenientes de plantas podadas.

En otro estudio (Uygun y Sari, 2000), se añadió un nuevo tratamiento donde se podó el tallo principal, pero se mantuvieron dos tallos secundarios. Los resultados reafirmaron que no existían diferencias significativas entre conservar o podar el tallo principal; sin embargo, si luego de la poda se conservaban dos tallos secundarios en lugar de solo uno, había un incremento de alrededor de un $20 \%$ en el rendimiento.

Otros investigadores, en Albania, analizaron el efecto de diferentes tipos de poda sobre el rendimiento y la calidad de fruta de melón tipo Galia (Jani y Hoxha, 2002). En esa investigación se utilizaron cinco tipos de poda: un tratamiento testigo sin poda; un tratamiento similar al testigo, pero con podas menores en los brotes; un tratamiento con poda del tallo principal y conservando un tallo secundario; un tratamiento con poda del tallo principal y conservando dos tallos secundarios; y un tratamiento con poda del tallo principal y manteniendo dos tallos secundarios, pero solo un tallo se entutoró y el otro se dejó crecer sobre el suelo. Los resultados demostraron que hubo un incremento significativo en la producción, en la cual se obtuvo el mejor rendimiento con el tratamiento que podaba el tallo principal y mantenía dos ejes. Además, se concluyó que, en mayor o menor medida, todos los tratamientos mejoraron el rendimiento, en comparación con el testigo sin poda.

En otro ensayo efectuado en Brasil, se utilizaron cuatro tipos de poda: un tallo con ramificaciones, un tallo sin ramificaciones, dos tallos, y cuatro tallos (Barni, Barni, y Silveira, 2003). Los datos obtenidos indicaron que al utilizar el sistema de dos tallos se obtenía el mejor rendimiento por área, así como el mejor peso por fruto, sin embargo, no se observaron diferencias importantes en la cantidad de frutos por planta. Es de resaltar que, en los sistemas con dos o cuatro tallos, la cosecha se atrasó en comparación con los sistemas de un solo tallo, indicando que la maduración y la cosecha de frutos son más tardíos cuando se mantiene una mayor cantidad de tallos por planta.

También en Brasil, otra investigación encontró que la productividad de dos híbridos de melón se incrementó debido al efecto de realizar poda en el tallo principal, en comparación con un tratamiento control sin poda (Pereira, Nogueira, Pedrosa, Negreiros, y Bezerra-Neto, 2003). El análisis no solo se limitó a la práctica de poda, sino también se observó el efecto de manejar varias densidades de siembra. Se concluyó que el número total de frutos comercializables se incrementó no solo debido a la poda, sino también al aumento en la densidad de siembra.

El objetivo de este trabajo fue determinar el efecto de dos densidades de siembra y dos sistemas de poda sobre el rendimiento y la calidad de melón tipo Amarillo cultivado en invernadero.

\section{Materiales y métodos}

El proyecto se llevó a cabo en Barrio San José de Alajuela, en la Estación Experimental Agrícola Fabio Baudrit Moreno (EEAFBM) de la Universidad de Costa Rica, ubicada a 883 m s. n. m. El cultivo se desarrolló en el invernadero del Programa de Hortalizas, y los análisis de calidad de fruta se llevaron a cabo en el Laboratorio de Ambientes Protegidos. El material utilizado fue el híbrido de melón tipo Amarillo JMX-904 F-1 (Cucumis melo L. var. inodorus), el cual presenta una planta andromonoica (Monge-Pérez, 2016).

El híbrido JMX-904 se sembró en almácigo el 7 demarzo de 2011, y se trasplantó el 4 de abril deese 
año. El trasplante se dio cuando las plántulas tenían al menos doshojas verdaderas desarrolladas.

Las plántulas fueron trasplantadas en sacos plásticos de $1 \mathrm{~m}$ de largo, $20 \mathrm{~cm}$ de ancho y 15 $\mathrm{cm}$ de alto, rellenos con aproximadamente 30 litros de fibra de coco. Previo al trasplante, los sacos se hidrataron y desinfectaron empleando el fungicida-bactericida Butrol (i.a. TCMTB), para lo cual se utilizó la dosis recomendada por el fabricante. Para evitar el anegamiento de los sacos, se realizaron dos cortes en la base de cada uno de ellos, aproximadamente a $1 \mathrm{~cm}$ de altura, que sirvieron como drenaje.

Para el manejo agronómico de las plantas, se le suplió un fertirriego diario con una solución nutritiva, especialmente diseñada para el cultivo de melón. El consumo fue de 0,5 a 1,5 L de agua con solución nutritiva por día, dependiendo del estado fenológico de la plantación, donde se dieron las mayores demandas hacia el final del ciclo del cultivo.

Se evaluaron dos densidades de siembra y dos sistemas de poda. La distancia entre hileras fue de 2,0 m, mientras que la distancia entre plantas fue de 33 y $25 \mathrm{~cm}$, equivalentes a una densidad de siembra de 1,5 y 2,0 plantas $/ \mathrm{m} 2$, respectivamente.

En cuanto a los sistemas de poda, se utilizaron los siguientes tratamientos: poda del tallo principal y manejo de un tallo secundario, y poda del tallo principal y manejo de dos tallos secundarios. La poda del tallo principal se realizó el 18 de abril de 2011, cuando las plantas tenían tres hojas verdaderas completamente desarrolladas (Gómez-Guillamón, Camero, y González-Fernández, 1997), a los 14 días después del trasplante (ddt), y se escogieron uno o dos tallos secundarios, según el tratamiento correspondiente.

Para todos los tratamientos, los tallos principales o secundarios fueron entutorados en una malla para el soporte de hortalizas, de polietileno, de 2,0 m de altura. En los tratamientos con poda, una vez entutorado el tallo secundario se realizaron podas semanales de la siguiente manera: los tallos terciarios que tenían fruto se podaron después de la segunda hoja emergida posterior al fruto; los tallos terciarios sin fruto se podaron después de la cuarta hoja emergida (Gómez-Guillamón, Camero, y González-Fernández, 1997). Esta poda de mantenimiento se hizo durante un periodo aproximado de tres semanas posteriores a la poda inicial; a partir de ahí, debido al escaso desarrollo de nuevos tallos terciarios, no fue necesario realizar más podas.

Con la aparición de las primeras flores en la plantación, se procedió a introducir una colmena de abejas (Apis mellifera), con el fin de promover una adecuada polinización.

El índice de cosecha se basó principalmente en la apariencia externa del fruto, en el cual se consideraron aptos para cosecha los frutos que tenían una coloración típica de madurez (color amarillo intenso).

La primera cosecha se produjo el 10 de junio de 2011 (67 ddt), la segunda se hizo el 22 de junio de ese año $(79 \mathrm{ddt})$, y la última se realizó el 1 de julio de 2011 (88 ddt). Se trasladaron los frutos al Laboratorio de Ambientes Protegidos de la EEAFBM inmediatamente después de cosechados, para su evaluación. Las variables evaluadas fueron: número total de frutos por planta, peso del fruto $(\mathrm{g})$, rendimiento total (ton/ha), porcentaje de sólidos solubles totales ( ${ }^{\circ}$ Brix), la resistencia a la penetración o firmeza de la pulpa $\left(\mathrm{kg} / \mathrm{cm}^{2}\right)$, y la relación pulpa:cavidad del fruto.

Cada fruto fue evaluado individualmente, iniciando por el peso, para lo cual se utilizó una balanza marca Ocony, modelo TH-I-EK, de 5000,0 $\pm 0,1 \mathrm{~g}$ de capacidad. Posteriormente, el fruto fue cortado longitudinalmente, y a una mitad se le evaluó el porcentaje de sólidos solubles totales, en tres sitios diferentes, empleando un refractómetro manual marca Atago, modelo N-1a, con 
una escala de $0,0-32,0 \pm 0,2{ }^{\circ}$ Brix. A la otra mitad se le midió la firmeza de la pulpa, también en tres sitios diferentes, y el grosor de la pulpa y de la cavidad del fruto; para la evaluación de la firmeza se utilizó un penetrómetro portátil marca Effegi, modelo FT-327 con una capacidad de $12,5 \pm 0,1 \mathrm{~kg} / \mathrm{cm} 2$, utilizando el puntero cuya base mide 7,5 mm de ancho. Para la medición del grosor de la pulpa y de la cavidad del fruto, una cinta métrica marca Assist, modelo 32G8025 , con una capacidad de $800,0 \pm 0,1 \mathrm{~cm}$. Con los datos individuales de grosor de pulpa y grosor de cavidad, se calculó la relación pulpa:cavidad. Adicionalmente, a partir de los datos de cosecha (número de frutos y peso de los mismos, por parcela) y de la densidad de siembra, se calculó el número total de frutos por planta y el rendimiento total (ton/ha).

Se utilizó un diseño experimental irrestricto al azar. Se evaluaron cuatro tratamientos (dos densidades de siembra y dos tipos de poda), cada uno con tres repeticiones. La parcela útil fue equivalente a dos metros lineales a lo largo de la hilera de siembra. La cantidad de plantas evaluadas varió en función de la densidad de siembra, en donde los tratamientos con la densidad de 1,5 plantas/m2 estuvieron conformados por seis plantas por repetición, y la densidad de 2,0 plantas/m2 por ocho plantas por repetición. Todos los frutos producidos dentro de cada parcela fueron recolectados y evaluados.

Para todas las variables, se realizó un análisis estadístico de varianza y se utilizó la prueba de LSD Fisher con una significancia de $5 \%$ para obtener las comparaciones entre tratamiento

\section{Resultados y discusión}

Para tener éxito en todo sistema de comercialización de productos agrícolas, es indispensable conocer el mercado meta y las características que ellos esperan en el producto final.
En Costa Rica, la cadena de comercialización del melón se ha adaptado a una ventana de exportación en el mercado internacional, la cual se basa en proveer de fruta a países del hemisferio norte durante su época de invierno, específicamente a Estados Unidos y Europa. Entre las características buscadas por cada mercado, además del tipo de melón, es de gran importancia el tamaño del fruto. Los consumidores estadounidenses tienden a preferir frutas más grandes, principalmente de tipo Cantaloupe, mientras que los europeos se inclinan por un melón pequeño e inodoro, características típicas de los melones tipo Honey Dew o Amarillo (Díaz-Porras y Sandí-Meza, 2007). Al analizar esto, cuando se usa un sistema de producción novedoso, como es la siembra de melón en invernadero en nuestro país, no solo es importante conocer un estimado de los rendimientos que se podrían obtener, sino que también es de vital importancia conocer qué tipo de fruto (tamaño) se puede conseguir de acuerdo con el sistema productivo que se piensa utilizar, para poder conocer si se cumplirá con lo exigido por el mercado meta.

Número de frutos por planta y peso promedio del fruto

Los datos de número total de frutos por planta y peso promedio del fruto se presentan en la tabla 1. Se observa que, para los cuatro tratamientos evaluados, no se encontraron diferencias significativas entre ellos en ninguna de estas variables, por lo tanto se puede afirmar que la poda o la densidad de siembra no generaron un efecto sobre ellas.

Se enfatiza que en el caso de la poda a dos tallos no se produjeron más frutos por planta, con respecto a la poda a un tallo. Dado que el costo de mano de obra de mantener dos tallos es mayor que el de mantener solo uno, parece que esta es una práctica que no se justifica económicamente. 
Tabla 1

Número total de frutos por planta y peso promedio del fruto, según cada tratamiento, para el melón Amarillo JMX-904

\begin{tabular}{|c|c|c|c|}
\hline \multicolumn{2}{|c|}{ Tratamiento } & \multirow[t]{2}{*}{ Número total de frutos por planta } & \multirow[t]{2}{*}{ Peso promedio del fruto $(\mathrm{g})$} \\
\hline $\begin{array}{l}\text { Densidad de siembra (número de } \\
\text { plantas } / \mathrm{m}^{2} \text { ) }\end{array}$ & $\begin{array}{l}\text { Tipo de poda (número de } \\
\text { tallos secundarios) }\end{array}$ & & \\
\hline \multirow[t]{2}{*}{1.5} & 1 & $2,08 \mathrm{a}$ & 511,4 a \\
\hline & 2 & $2,00 \mathrm{a}$ & 530,4 a \\
\hline \multirow{2}{*}{2.0} & 1 & $2,58 \mathrm{a}$ & 530,8 a \\
\hline & 2 & $2,08 \mathrm{a}$ & $533,0 \mathrm{a}$ \\
\hline
\end{tabular}

Nota: elaboración propia, a partir de los datos obtenidos. Letras distintas indican diferencias significativas ( $\mathrm{p} \leq 0,05)$, según la prueba LSD Fisher.

También se debe anotar que el tamaño del fruto de este híbrido de melón es pequeño (peso promedio del fruto entre 511-533 g), lo que contrasta con el peso normal en el mercado nacional de melón Cantaloupe en Costa Rica (entre 1,0 y 2,0 kg). En condiciones de producción a campo abierto en Guanacaste y Puntarenas, Costa Rica, este híbrido produce frutos con un peso mayor, entre 800 y 1660 g (Monge-Pérez, datos sin publicar).

En otros ensayos llevados a cabo en el invernadero de la EEAFBM, el híbrido JMX-904 presentó un peso promedio del fruto entre $268,7 \mathrm{y}$ $338,6 \mathrm{~g}$, al ser cultivado a una densidad de 2,6 plantas/m2 (Autor2, 2016). Esta tendencia a obtener frutos de menor peso con una densidad mayor ya había sido informada por otros investigadores (Nerson, 1999; Rodríguez, Shaw y Cantliffe, 2007). Con respecto a la producción por planta, en dichos ensayos en la EEAFBM este híbrido produjo entre 3,75 y 4,75 frutos por planta (Monge-Pérez, 2016); lo cual indica que en esos ensayos se presentó un mayor cuajado de frutos, pero estos fueron de menor tamaño; en esa oportunidad, se dejó crecer la planta a libre crecimiento, por lo que seguramente se presentaron más de dos tallos por planta. En este sentido, parece que la regulación del número de tallos por planta sí es importante en este híbrido para obtener frutos de mayor peso, y por lo tan- to, mayor calidad comercial, tal y como sucedió en la presente investigación.

\section{Rendimiento total}

Los datos de rendimiento total se presentan en la figura 1. Los valores muestran una clara tendencia a obtener un mayor rendimiento total conforme se incrementa la densidad de siembra. El rendimiento obtenido con la densidad de 2,0 plantas $/ \mathrm{m}^{2}$, con poda a un tallo secundario, mostró el mayor valor ( 27,5 ton/ha), y este resultado fue estadísticamente diferente a los valores que se obtuvieron con la densidad de 1,5 plantas/m2, tanto con poda a uno como a dos tallos secundarios (15,8 y 15,1 ton/ha, respectivamente).

Al comparar el rendimiento total obtenido para ambos tipos de poda, no se encuentran diferencias entre ellos, dentro de cada una de las densidades de siembra. Este resultado es distinto al informado por Uygun y Sari (2000), quienes obtuvieron un $20 \%$ más de rendimiento con la poda a dos tallos secundarios que con la poda a un tallo secundario. Por lo tanto, las diferencias encontradas en el rendimiento total en el presente ensayo se deben únicamente al factor densidad de siembra.

Diversos estudios presentan resultados similares a los obtenidos para el melón JMX-904. En Croacia y Florida, el aumento en la densidad de siembra generó un incremento en el rendimiento 
Rendimiento (ton/ha)

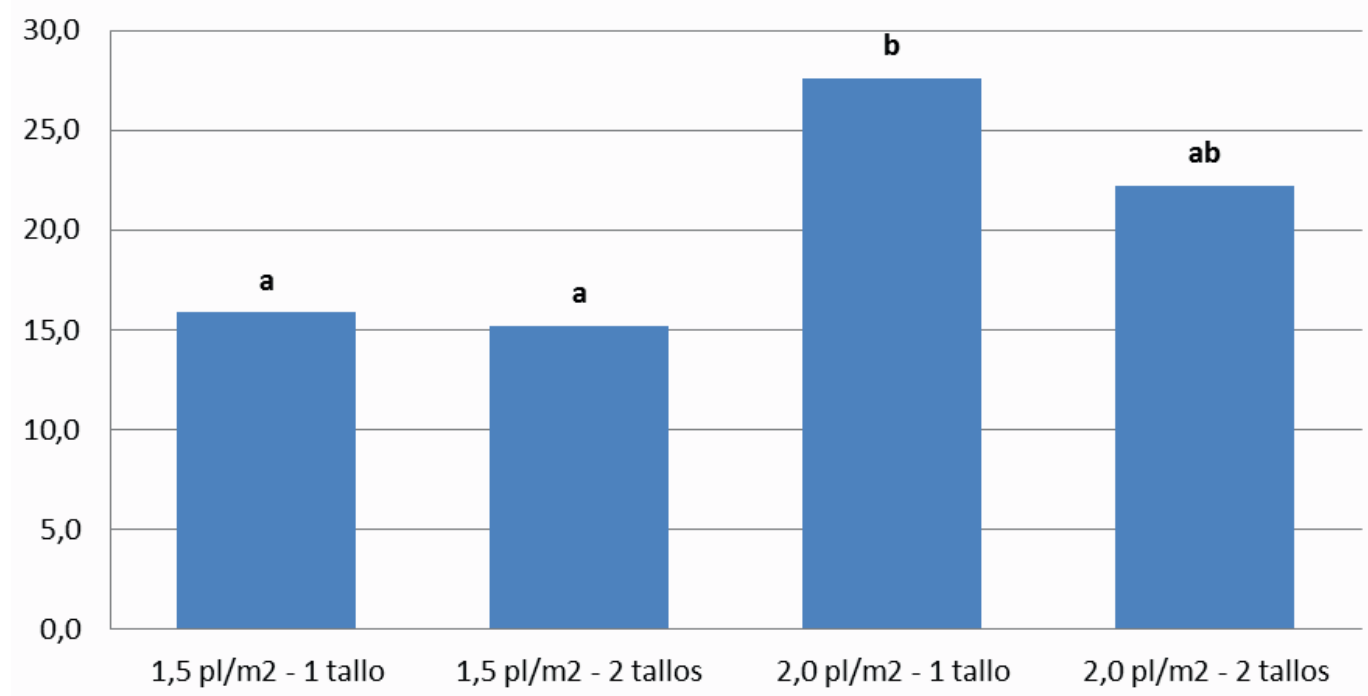

Figura 1. Rendimiento total (ton/ha), según cada tratamiento, para el melón Amarillo JMX-904.

Nota: elaboración propia a partir de los datos obtenidos. Letras distintas indican diferencias significativas $(p \leq .05)$, según la prueba LSD Fisher.

final, sin afectar el peso promedio de los frutos (Ban, Goreta y Borosic, 2006; Rodríguez, Shaw y Cantliffe, 2007).

En otros ensayos realizados en el invernadero de la EEAFBM, el híbrido JMX-904 mostró un rendimiento total de entre 32,98 y 33,15 ton/ha (Monge-Pérez, 2016) con una densidad de siembra de 2,6 plantas/m2. Por lo tanto, ese mayor rendimiento obtenido en esos ensayos con respecto a los encontrados en el presente estudio, podría deberse al uso de una mayor densidad de siembra, efecto que ha sido informado por otros investigadores (Nerson, 1999; Pereira, Nogueira, Pedrosa, Negreiros y Bezerra-Neto, 2003; Ban, Goreta y Borosic, 2006; Rodríguez, Shaw y Cantliffe, 2007).

En varios estudios que involucraron el uso de sistemas de podas, donde se eliminaba el tallo principal y se manejaban uno o varios tallos secundarios, se ha documentado que esta práctica mejoró el rendimiento final obtenido, a causa de una mejor distribución de los recursos de la planta, pues la poda elimina el exceso de vigor vegetativo y genera un mejor equilibrio con la parte productiva (Jani y Hoxha, 2002; Barni, Barni y Silveira, 2003; Pereira, Nogueira, Pedrosa, Negreiros y Bezerra-Neto, 2003). Otras investigaciones revelaron que el rendimiento final no siempre se ve mejorado por el uso de podas, sino que pueden ser otras características de calidad las que se vean afectadas (Eltez, Tüzel y Boztok, 1999; Uygun y Sari, 2000). Para el híbrido analizado en el presente ensayo, se observó que el tipo de poda utilizado no mejoró el rendimiento final obtenido, sino que esta variable parece ser más dependiente de la densidad utilizada.

\section{Calidad del fruto}

El término calidad puede ser difícil de definir porque involucra la opinión de diferentes personas acerca del estado de un producto determinado. Lo que un mercado meta específico define como un fruto de calidad no necesariamente será igual al concepto de calidad de otro mercado. Ante esta variabilidad, la definición váli- 
da de calidad sería aquel estado de un producto que logra satisfacer las expectativas del cliente o consumidor final (Hewett, 2006).

La calidad de un fruto está compuesta de varios atributos o componentes: intrínsecos o propios del producto (forma, color, textura, dulzura), y extrínsecos (sistemas de producción y canales de distribución utilizados, entre otros aspectos comerciales). El sistema de producción elegido tiene la capacidad de afectar los atributos intrínsecos de calidad, por lo que es importante determinar el efecto que tiene realizar alguna práctica agrícola específica sobre la calidad final del producto. Los atributos extrínsecos en realidad no están tan ligados con la calidad propia del fruto, sino que están más relacionados con la decisión de compra del consumidor, por ejemplo, cuando hay un favoritismo hacia productos orgánicos versus convencionales (Calvo, 2005; Hewett, 2006).

Los componentes intrínsecos de calidad que son importantes en el melón son: el porcentaje de sólidos solubles totales, la firmeza de la pulpa, y la relación pulpa:cavidad. El primero hace referencia a la dulzura y el sabor de la fruta, y es posiblemente el más importante de los tres. La firmeza está más ligada a la capacidad de resistencia del melón para mantenerse adecuadamente durante el periodo poscosecha, aunque también es importante para obtener un fruto crujiente, característica deseada por algunos mercados. Por último, la relación pulpa:cavidad hace referencia a la cantidad de pulpa comestible y la cantidad de espacio interno que no es aprovechable para el consumidor final; lo ideal es que esta relación sea cercana a 1, esto indica que el espacio interno entre la pulpa y la cavidad son equitativos.

En nuestro país, no están claramente definidos los valores mínimos en los índices de calidad para considerar los frutos de melón como comercializables o no. Para el porcentaje de sólidos solubles totales, se ha tomado como válido el valor de la norma de California, la cual indica que los frutos de melón deberían tener un mínimo de 10,0 ${ }^{\circ}$ Brix para ser comercializables (Sáenz, 2005) sin importar el tipo de melón evaluado. Lo mismo ocurre para la firmeza de pulpa y la relación pulpa:cavidad, cuyos valores aceptados son de conocimiento propio del gremio melonero nacional, pero no existe ninguna institución local que los haya definido como valores oficiales.

En la tabla 2, se muestran los valores de porcentaje de sólidos solubles totales, firmeza de la pulpa y relación pulpa:cavidad para el melón JMX-904, para cada uno de los tratamientos utilizados. No se presentaron diferencias significativas entre ninguno de los tratamientos para estas tres variables.

Los valores de porcentaje de sólidos solubles totales están por encima de $10,0^{\circ}$ Brix, que es el mínimo requerido para que el fruto se considere comercializable y sea aceptado en la mayoría de los mercados (Sáenz, 2005). Los resultados obtenidos para esta variable con el melón JMX-904 son extraordinariamente buenos, por lo que esta variedad podría representar una opción productiva interesante para los agricultores que buscan un producto diferenciado para un nicho específico de mercado al explotar dicha cualidad.

En otros ensayos realizados en el invernadero de la EEAFBM con el híbrido JMX-904, se obtuvieron valores entre 14,7 y 16,4 ${ }^{\circ}$ Brix para esta variable (Monge-Pérez, 2016).

Diversas investigaciones han mostrado resultados contrastantes con respecto al efecto de la densidad de siembra o la poda sobre el porcentaje de sólidos solubles totales. En melones tipo Galia, no se encontraron diferencias significativas en esta variable cuando se evaluaron densidades de 1,7 hasta 4,1 plantas/m² (Rodríguez, Shaw y Cantliffe, 2007). Por otro lado, investigaciones con melones tipo Cantaloupe mostraron 
Tabla 2

Porcentaje de sólidos solubles totales, firmeza de la pulpa y relación pulpa: cavidad del fruto, según cada tratamiento, para el melón Amarillo JMX-904

\begin{tabular}{|c|c|c|c|c|}
\hline \multicolumn{2}{|c|}{ Tratamiento } & \multirow{2}{*}{$\begin{array}{l}\text { Porcentaje de sólidos solu- } \\
\text { bles totales ( }{ }^{\circ} \text { Brix) }\end{array}$} & \multirow{2}{*}{$\begin{array}{l}\text { Firmeza de la } \\
\text { pulpa }\left(\mathrm{kg} / \mathrm{cm}^{2}\right)\end{array}$} & \multirow{2}{*}{$\begin{array}{l}\text { Relación pulpa: } \\
\text { cavidad del fruto }\end{array}$} \\
\hline $\begin{array}{l}\text { Densidad de siembra (número de } \\
\text { plantas } / \mathrm{m}^{2} \text { ) }\end{array}$ & $\begin{array}{l}\text { Tipo de poda (número } \\
\text { de tallos secundarios) }\end{array}$ & & & \\
\hline \multirow[t]{2}{*}{1.5} & 1 & $14,2 \mathrm{a}$ & $2,41 \mathrm{a}$ & $1,15: 1 \mathrm{a}$ \\
\hline & 2 & 14,6 a & $2,52 \mathrm{a}$ & $1,26: 1 \mathrm{a}$ \\
\hline \multirow{2}{*}{2.0} & 1 & $14,7 \mathrm{a}$ & $2,28 \mathrm{a}$ & $1,11: 1 \mathrm{a}$ \\
\hline & 2 & $15,0 \mathrm{a}$ & $2,51 \mathrm{a}$ & $1,09: 1 \mathrm{a}$ \\
\hline
\end{tabular}

Nota: elaboración propia a partir de los datos obtenidos. Letras distintas indican diferencias significativas $(p \leq .05)$, según la prueba LSD Fisher.

que, conforme aumenta la densidad de siembra, se produjo una disminución en el porcentaje de sólidos solubles totales del fruto (Nerson, 2002; García, Rodríguez, Lugo y Rodríguez, 2009). Sin embargo, parece que el tipo de melón (genotipo) y las condiciones climáticas del lugar donde se cultive influyen enormemente sobre la variable porcentaje de sólidos solubles totales del fruto, $\mathrm{y}$ tienen un mayor impacto que los tratamientos de poda o densidad de siembra.

Con respecto a la firmeza de la pulpa en los frutos de melón, en el país no se ha definido un valor mínimo aceptable para esta variable, pero en fincas productoras y exportadoras se considera como valor óptimo $2,0 \mathrm{~kg} / \mathrm{cm} 2$ o más (Monge-Pérez, 2011). Según este parámetro, todos los tratamientos superan el valor mínimo necesario de firmeza para ser comercializables, por lo tanto se consideran satisfactorios, pues no necesariamente hay preferencia en el mercado por frutas con alta firmeza, sino que solo es importante que sobrepasen el mínimo necesario.

En otros ensayos llevados a cabo en el invernadero de la EEAFBM, el híbrido JMX-904 obtuvo valores de firmeza de pulpa de $2,1 \mathrm{~kg} / \mathrm{cm} 2$ (Monge-Pérez, 2016).

En cuanto a la relación pulpa:cavidad, todos los valores fueron mayores a 1 , de tal manera, se concluye que los frutos poseen una adecuada cantidad de pulpa comestible, lo que confirma otra buena característica de la calidad interna del fruto de este híbrido de melón.

Los tratamientos de poda y densidad evaluados no provocaron un efecto importante sobre la calidad, pues siempre se obtuvo una calidad de fruta aceptable desde el punto de vista comercial.

\section{Conclusiones y recomendaciones}

No se presentaron diferencias significativas entre los tratamientos evaluados, para las variables peso promedio del fruto, número total de frutos por planta, porcentaje de sólidos solubles totales, firmeza de pulpa y relación pulpa:cavidad del fruto, para el melón Amarillo JMX-904.

Los resultados indican que el tipo de poda no afectó el rendimiento, pero la densidad de siembra sí mejoró la producción total. El tratamiento con la mayor densidad de siembra (2,0 plantas/ m2) y con poda a un tallo secundario fue el que presentó el mejor rendimiento (27,5 ton/ha), y este resultado fue significativamente superior con respecto a los dos tratamientos con la densidad de siembra de 1,5 plantas $/ \mathrm{m}^{2}$. Se concluye que con una mayor densidad de plantas por área, se logra un mejor rendimiento, sin generar diferencias en el peso promedio de los frutos.

A pesar de que el tamaño del fruto del melón JMX-904 es pequeño (peso promedio del fruto 
entre 511-533 g), la muy buena calidad del mismo (especialmente el alto porcentaje de sólidos solubles totales, entre $14,2-15,0{ }^{\circ}$ Brix), podría hacer de este híbrido una opción productiva interesante para los agricultores que buscan un producto diferenciado para un nicho de mercado específico.

Se recomienda realizar otras investigaciones sobre este tema, en las que se incluyan otras densidades de siembra y otros tipos de poda, y se evalúen tanto aspectos de rendimiento y calidad como de costos de producción, con el fin de desarrollar sistemas productivos adaptados a las condiciones del país.

\section{Agradecimientos}

Los autores agradecen la colaboración de Cristina Arguedas, Julio Vega y Carlos González en el trabajo de campo, y de Mario Monge en la traducción del resumen al idioma inglés. Asimismo, agradecen el financiamiento recibido por parte de la Vicerrectoría de Investigación de la Universidad de Costa Rica para la realización de este trabajo.

\section{Referencias}

Alvarado-Sánchez, T. y Monge-Pérez, J. (2015). Efecto de la aplicación de bioactivadores y del raleo manual de frutos sobre el rendimiento y la calidad de melón (Cucumis melo L.) bajo cultivo protegido en Costa Rica. Tecnología en Marcha, 28 (4), Octubre-Diciembre, 15-25.

Ban, D., Goreta, S., y Borosic, J. (2006). Plant spacing and cultivar affect melon growth and yield components. Scientia Horticulturae, 109, 238-243.

Barni, V., Barni, N. A., y Silveira, J. R. (2003). Meloeiro em estufa: duas hastes éo melhor sistema de condução. Ciência Rural, 33 (6), 1039-1043.

Calvo, P. (2005). Sistemas poscosecha: generalidades. En G. Meléndez, y G. Umaña, Sistemas poscosecha en frutas de mango, melón y sandía: conceptos y aplicaciones (págs. 1-20). San José, Costa Rica: INTA.

Díaz-Porras, R., y Sandí-Meza, V. (2007). La cadena de melón en Costa Rica: potencialidades y desafíos internacionales. Revista Centroamericana de Ciencias Sociales, 4(2), 60-101.

Eltez, R. Z., Tüzel, Y., y Boztok, K. (1999). Effects of different growing media and pruning methods on greenhouse muskmelon production. Acta Horticulturae, 491, 363-368.

FAO (2002). El cultivo protegido en clima mediterráneo. Roma, Italia: FAO.

FAO (2012). Información sobre el cultivo de melón. Recuperado de http://faostat.fao.org.

García, J. C., Rodríguez, G., Lugo, J. G., y Rodríguez, V. (2009). Efecto del cultivar y distancia entre plantas sobre características físico-químicas del fruto del melón (Cucumis melo L.). Revista de la Facultad de Agronomía (LUZ), 26, 141-158.

Gómez-Guillamón, M. L., Camero, R., y González-Fernández, J. (1997). El melón en invernadero. En A. Namesny, Melones (págs. 67-77). Barcelona, España: Ediciones de Horticultura S. L.

Hewett, E. (2006). An overview of preharvest factors influencing postharvest quality of horticultural products. International Journal of Postharvest Technology and Innovation, 1(1), 4-15.

Jani, S., y Hoxha, S. (2002). The effect of plant pruning on production of melon grown under PVC greenhouse conditions. Acta Horticulturae, 579, 377-381.

Monge Perez, J. (2011). Aspectos económicos en la producción de melón. Alajuela, Costa Rica: Universidad de Costa Rica.

Monge Perez, J. (2014). Producción y exportación de melón (Cucumis melo) en Costa Rica. Tecnología en Marcha, 27(1), 93-103. 
Monge Perez, J. (2016). Evaluación de 70 genotipos de melón (Cucumis melo L.) cultivados bajo invernadero en Costa Rica. Intersedes, 17(36), 2-41.

National Research Council. (2008). Lost crops of Africa. Volume III: Fruits. Washington D. C.: The National Academic Press.

Nerson, H. (1999). Effects of population density on fruit and seed production in muskmelons. Acta Horticulturae, 492, 65-70.

Nerson, H. (2002). Relationship between plant density and fruit and seed production in muskmelon. Journal of the American Society for Horticultural Science, 127(5), 855-859.

Pereira, F. H., Nogueira, I., Pedrosa, J., Negreiros, M., y Bezerra-Neto, F. (2003). Poda da haste principal e densidade de cultivo na produção e qualidade de frutos em híbridos de melão. Horticultura Brasileira, 21(2), 191-196.

Reche, J. (1995). Poda de hortalizas en invernadero (calabacín, melón, pepino y sandía). Madrid, España: Ministerio de Agricultura, Pesca y Alimentación.

Reche, J. (2007). Cultivo intensivo de melón. Madrid, España: Ministerio de Agricultura, Pesca y Alimentación.

Rodríguez, J., Shaw, N., y Cantliffe, D. (2007). Influence of plant density on yield and fruit quality of greenhouse-grown galia muskmelons. Hort Technology, 17 (4), 580-585.
Sáenz, M. V. (2005). Biología y fisiología de los productos frescos. En G. Meléndez, y G. Umaña, Sistemas poscosecha en frutas de mango, melón y sandía: conceptos y aplicaciones (págs. 64-90). San José, Costa Rica: INTA.

Santos, B. M., Obregón-Olivas, H. A., y Salamé-Donoso, T. P. (2010). Producción de hortalizas en ambientes protegidos: estructuras para la agricultura protegida. Wimauma, Florida, EE. UU.: University of Florida.

SEPSA (2010). Boletín estadístico agropecuario No. 20, serie cronológica 2006-2009. San José, Costa Rica: Secretaría Ejecutiva de Planificación Sectorial Agropecuaria.

SEPSA. (2012). Boletín estadístico agropecuario No. 22, serie cronológica 2008-2011. San José, Costa Rica: Secretaría Ejecutiva de Planificación Sectorial Agropecuaria.

Torres, J. (1997). Los tipos demelón comerciales. En A. Namesny, Melones (págs. 13-20). Barcelona, España: Ediciones de Horticultura S.

Uygun, N., y Sari, N. (2000). The effects of different pruning methods and height of fruit setting on plant growth, yield and fruit quality of melons grown in greenhouses. Turkish Journal of Agriculture and Forestry, 24(1), 365-373.

Recibido: : 4 de enero de 2017

Aceptado: 15 de marzo de 2017 